\title{
Nonlinear Klein-Gordon and Schrödinger Equations by the Projected Differential Transform Method
}

\author{
Younghae Do ${ }^{1}$ and Bongsoo Jang ${ }^{2}$ \\ ${ }^{1}$ Department of Mathematics, Kyungpook National University, Daegu 702-701, Republic of Korea \\ ${ }^{2}$ Ulsan National Institute of Science and Technology (UNIST), \\ Ulsan Metropolitan City 689-798, Republic of Korea
}

Correspondence should be addressed to Bongsoo Jang, bsjang@unist.ac.kr

Received 12 April 2012; Accepted 21 June 2012

Academic Editor: Elena Litsyn

Copyright (C) 2012 Y. Do and B. Jang. This is an open access article distributed under the Creative Commons Attribution License, which permits unrestricted use, distribution, and reproduction in any medium, provided the original work is properly cited.

The differential transform method (DTM) is based on the Taylor series for all variables, but it differs from the traditional Taylor series in calculating coefficients. Even if the DTM is an effective numerical method for solving many nonlinear partial differential equations, there are also some difficulties due to the complex nonlinearity. To overcome difficulties arising in DTM, we present the new modified version of DTM, namely, the projected differential transform method (PDTM), for solving nonlinear partial differential equations. The proposed method is applied to solve the various nonlinear Klein-Gordon and Schrödinger equations. Numerical approximations performed by the PDTM are presented and compared with the results obtained by other numerical methods. The results reveal that PDTM is a simple and effective numerical algorithm.

\section{Introduction}

The solutions of linear and nonlinear partial differential equations play an important role in many fields of science and engineering such as solid-state physics, nonlinear optics, plasma physics, fluid dynamics, chemical kinetics, and biology. In this work, we consider two nonlinear partial differential equations. One is the Klein-Gordon equation with power nonlinearity:

$$
u_{t t}+\alpha u_{x x}+\beta u+\gamma u^{n}=f,
$$


with the constant $n=2$ or 3 , and another is the nonlinear Schrödinger equation:

$$
i u_{t}+\alpha \Delta u+\beta u \psi+\gamma|u|^{2} u=0
$$

with a trapping potential $\psi$ and $i^{2}=-1$. Here, $\alpha, \beta$, and $\gamma$ are real constants and $\Delta u=u_{x x}+u_{y y}$. For solving nonlinear partial differential equations including, above nonlinear problems, many powerful methods have been developed such as Bäcklund the transformation [1], Darboux's transformation [2,3], Tanh function [4], homogeneous balance [5], Jacobi's elliptic method [6, 7], F-expansion method [8,9], and auxiliary equation [10-12]. Recently, iterativetype methods such as Adomian decomposition [13-16], homotopy perturbation [17-20], and variational iteration [21-24] have been used to find accurate approximations by using symbolic mathematical packages: Mathematica, Maple, and Matlab. For solving nonlinear Schrödinger equations, many efficient discretized numerical schemes have been proposed such as split-step finite-difference method (SSFD) [25, 26], split-step Fourier pseudospectral method [25], pseudospectral method based on Hermit functions [26], and the method [27] in one-dimensional problems.

Here, we propose the differential transform method to solve our model problems in (1.1), (1.2). The DTM is close to the Taylor series, but it is different from the conventional high-order Taylor series in determining coefficients. The basic idea of DTM was introduced by Zhou [28] in solving initial value problems in electrical circuit analysis. The DTM has been employed to solve many important problems science and engineering fields and obtain highly accurate approximations [28-39]. However, it also have some difficulties due to the nonlinearity. Here, we introduce the modified version of the standard DTM, the projected DTM, which is a simple and effective method comparing with the standard DTM.

This paper is organized as follows. A detail description of the projected DTM will be given in Section 2. To our model problems, nonlinear Klein-Gordon and Schrödinger equations, both the standard DTM and the projected DTM, are applied and the corresponding algebraic equations are presented in Section 3. In Section 4, various numerical examples are demonstrated. For each illustrative example, numerical results obtained by DTM, PDTM, and other numerical method are compared. The conclusion will be made in the last section.

\section{Description of the Projected Differential Transform Method}

In this section, we describe the definition and some properties of the standard DTM. Moreover, we present the basic idea of the projected differential transform method. Suppose a function $w(x, y, t)$ is analytic in the given domain $T$ and $\left(x_{0}, y_{0}, t_{0}\right) \in T$. Let us define the differential transform $W(k, h, m)$ of $w(x, y, t)$ at $\left(x_{0}, y_{0}, t_{0}\right)$ by

$$
W(k, h, m)=\frac{1}{k ! h ! m !}\left[\frac{\partial^{k+h+m} w(x, y, t)}{\partial x^{k} \partial y^{h} \partial t^{m}}\right]_{x=x_{0}, y=y_{0}, t=t_{0}}
$$

The differential inverse transform of $W(k, h, m)$ is defined by

$$
w(x, y, t)=\sum_{k=0}^{\infty} \sum_{h=0}^{\infty} \sum_{m=0}^{\infty} W(k, h, m)\left(x-x_{0}\right)^{k}\left(y-y_{0}\right)^{h}\left(t-t_{0}\right)^{m}
$$


For $\left(x_{0}, y_{0}, t_{0}\right)=(0,0,0)$, we have

$$
w(x, y, t)=\sum_{k=0}^{\infty} \sum_{h=0}^{\infty} \sum_{m=0}^{\infty} \frac{1}{k ! h ! m !}\left[\frac{\partial^{k+h+m} w(x, y, t)}{\partial x^{k} \partial y^{h} \partial t^{m}}\right]_{x=0, y=0, t=0} x^{k} y^{h} t^{m}
$$

In other words,

$$
w(x, y, t)=\sum_{k=0}^{\infty} \sum_{h=0}^{\infty} \sum_{m=0}^{\infty} W(k, h, m) x^{k} y^{h} t^{m}
$$

Some fundamental operations for the standard DTM are presented in Table 1. It has been proved that the standard DTM is an efficient tool for solving many linear and nonlinear problems [28-39]. However, there are also some difficulties in DTM. Let us consider the differential transform for $u^{3}$ which involves six summations in the Table 1 . Thus, it is necessary to have a lot of computational work to calculate such differential transform $U(k, h, m)$ for the large numbers $k, h, m$.

In what follows, we introduce the basic idea of modified version of the DTM, the projected DTM. The DTM is based on the Taylor series for all variables. Here, we consider the Talyor series of the function $u$ with respect to the specific variable. Assume that the specific variable is the variable $t$. Then we have the Taylor series expansion of the function $u$ at $t=t_{0}$ as follows:

$$
u(x, y, t)=\sum_{m=0}^{\infty} \frac{1}{m !}\left[\frac{\partial^{m}}{\partial t^{m}} u(x, y, t)\right]\left(t-t_{0}\right)^{m}
$$

Definition 2.1. The projected differential transform $U(x, y, m)$ of $u(x, y, t)$ with respect to the variable $t$ at $t_{0}$ is defined by

$$
U(x, y, m)=\frac{1}{m !}\left[\frac{\partial^{m}}{\partial t^{m}} u(x, y, t)\right]_{t=t_{0}} .
$$

Definition 2.2. The projected differential inverse transform of $U(x, y, m)$ with respect to the variable $t$ at $t_{0}$ is defined by

$$
u(x, y, t)=\sum_{m=0}^{\infty} U(x, y, m)\left(t-t_{0}\right)^{m}
$$

Since the PDTM results from the Taylor series of the function with respect to the specific variable, it is expected that the corresponding algebraic equation from the given problem is much simpler than the result obtained by the standard DTM. The detail description of the corresponding algebraic equation will be followed in the next section. 
Table 1: Fundamental operations for the three-dimensional DTM.

\begin{tabular}{ll}
\hline $\begin{array}{l}\text { Original function } \\
w(x, y, t)\end{array}$ & Transformed function $W(k, h, m)$ \\
\hline$u(x, y, t) \pm v(x, y, t)$ & $U(k, h, m) \pm V(k, h, m)$ \\
$c u(x, y, t)$ & $c U(k, h, m)$ \\
$\frac{\partial^{r+s+p} u(x, y, t)}{\partial x^{r} \partial y^{s} \partial t^{p}}$ & $\frac{(k+r) !}{k !} \frac{(h+s) !}{s !} \frac{(m+p) !}{p !} U(k+r, h+s, m+p)$ \\
$\frac{\partial u(x, y, t)}{\partial x} \frac{\partial v(x, y, t)}{\partial y}$ & $\sum_{r=0}^{k} \sum_{s=0}^{h} \sum_{p=0}^{m}(k-r+1)(h-s+1) U(k-r+1, s, p) U(r, h-s+1, m-p)$ \\
$u(x, y, t) v(x, y, t)$ & $\sum_{r=0}^{j} \sum_{s=0}^{h} \sum_{p=0}^{m} U(r, h-s, m-p) V(k-r, s, p)$ \\
$u(x, y, t) v(x, y, t) g(x, y, t)$ & $\sum_{r=0}^{k} \sum_{\tau=0}^{k-r} \sum_{s=0}^{h} \sum_{p=0}^{h-s} \sum_{l=0}^{m} \sum_{q=0}^{m-l} U(r, h-s-p, l) V(\tau, s, m-l-q) G(k-r-\tau, p, q)$ \\
\hline
\end{tabular}

\section{Comparison of the Standard and Projected DTMs}

In this section, we present the comparison of the standard DTM and the projected DTM for solving our model problems, the nonlinear Klein-Gordon and Schrödinger equations. As seen in the previous section, it is the key to obtain the corresponding algebraic equation of the differential transform for the given problems in DTM. For the model problems, we present the corresponding algebraic equations of the differential transform in the standard DTM and the projected DTM at $(x, y, t)=(0,0,0)$. Firstly, let us consider the following one-dimensional nonlinear Klein-Gordon equation:

$$
u_{t t}+\alpha u_{x x}+\beta u+\gamma u^{n}=f(x, t), \quad x \in(a, b),
$$

with initial conditions

$$
u(x, 0)=g_{1}(x), \quad u_{t}(x, 0)=g_{2}(x),
$$

where $\alpha, \beta$, and $\gamma$ are known constants and the constant $n=2$ or 3 . The standard DTM for the (3.1) gives the following algebraic equation:

$$
\begin{gathered}
(h+1)(h+2) U(k, h+2)+\alpha(k+1)(k+2) U(k+2, h) \\
+\beta U(k, h)+\gamma G(k, h)=F(k, h),
\end{gathered}
$$

where

$$
G(k, h)= \begin{cases}\sum_{r=0}^{k} \sum_{s=0}^{h} U(r, h-s) U(k-r, s) & n=2 \\ \sum_{r=0}^{k} \sum_{\tau=0}^{k-r} \sum_{s=0}^{h} \sum_{p=0}^{h-s} U(r, h-s-p) U(\tau, s) U(k-r-\tau, p) & n=3,\end{cases}
$$


and $F(k, h)$ is the differential transform for the function $f(x, y)$. The initial conditions give $U(k, 0)=G_{1}(k), U(k, 1)=G_{2}(k)$, where $G_{1}(k), G_{2}(k)$ are the differential transforms for the function $g_{1}(x), g_{2}(x)$, respectively.

The projected DTM with respect to variable $t$ gives the following algebraic equation:

$$
(h+1)(h+2) U(x, h+2)+\alpha U_{x x}(x, h)+\beta U(x, h)+\gamma G(x, h)=F(x, h),
$$

where

$$
G(x, h)= \begin{cases}\sum_{s=0}^{h} U(x, h-s) U(x, s) & n=2 \\ \sum_{s=0}^{h} \sum_{p=0}^{h-s} U(x, h-s-p) U(x, s) U(x, p) & n=3\end{cases}
$$

and $F(x, h)$ is the projected differential transform of $f(x, t)$ with respect to the variable $t$. The initial conditions give $U(x, 0)=g_{1}(x)$ and $U(x, 1)=g_{2}(x)$.

Here, we apply the DTM to solve the following Schrödinger equation:

$$
i u_{t}+\alpha \Delta u+\beta u \psi+\gamma|u|^{2} u=0
$$

with an initial condition $u(x, y, 0)=g(x, y)$. Then the standard DTM gives the following

$$
\begin{aligned}
i U(k, h, m+1) & +\alpha[(k+1)(k+2) U(k+2, h, m)+(h+1)(h+2) U(k, h+2, m)] \\
& +\beta \sum_{r=0}^{k} \sum_{s=0}^{h} \sum_{p=0}^{m} U(r, h-s, m-p) \Psi(k-r, s, p) \\
& +\gamma \sum_{r=0}^{k} \sum_{\tau=0}^{k-r} \sum_{s=0}^{h} \sum_{p=0}^{h-s} \sum_{l=0}^{m} \sum_{q=0}^{m-l} U(r, h-s-p, l) \\
& \times \overline{U(\tau, s, m-l-q)} U(k-r-\tau, p, q)=0,
\end{aligned}
$$

with $U(k, h, 0)=G(k, h) ; G(k, h)$ is the differential transform of $g(x, y)$.

The projected DTM with respect to the variable $t$ gives the following algebraic equation:

$$
\begin{aligned}
i(m+1) U(x, y, m+1) & +\alpha\left(U_{x x}+U_{y y}\right)(x, y, m)+\beta \sum_{p=0}^{m} U(x, y, m-p) \Psi(x, y, p) \\
& +\gamma \sum_{l=0}^{m} \sum_{q=0}^{m-l} U(x, y, m-l-q) \overline{U(x, y, l)} U(x, y, q)=0
\end{aligned}
$$

where $\Psi$ is the differential transform for the trapping potential function $\psi$ and $U(x, y, 0)=$ $g(x, y)$. 


\section{Illustrative Examples}

In order to show the effectiveness of the PDTM for solving the nonlinear Klein-Gordon and Schrödinger equations, several examples are demonstrated. For all illustrative examples, we consider the projected differential transform with respect to the variable $t$. To compare with numerical results obtained by DTM and PDTM, we define the partial sum of both methods as follows:

$$
\begin{gathered}
S_{p, q, r}^{\mathrm{dtm}}=\sum_{k=0}^{p} \sum_{h=0}^{q} \sum_{m=0}^{r} U(k, h, m) x^{k} y^{h} t^{m}, \\
S_{r}^{\mathrm{pdtm}}=\sum_{m=0}^{r} U(x, y, m) t^{m} .
\end{gathered}
$$

Example 4.1. Let us consider the nonlinear Klein-Gordon equation (3.1) with quadratic nonlinearity $n=2$ with constants $\alpha=-1, \beta=0$, and $\gamma=1$ in the interval $(0,1)$ [35]. The initial conditions are given by

$$
u(x, 0)=1+\sin x, \quad u_{t}(x, 0)=0 .
$$

That is, $g_{1}(x)=1+\sin x$ and $g_{2}(x)=0$ in (3.2).

The Standard DTM. Using initial conditions $g_{i}(x), i=1,2$ yields the following differential transforms $U(k, 0), U(k, 1), k=0,1,2, \ldots$ :

$$
\begin{aligned}
& U(1,0)=1, \quad U(2 k+1,0)=\frac{(-1)^{k}}{(2 k+1) !} \\
& U(2 k, 0)=0, \quad U(k, 1)=0 .
\end{aligned}
$$

Substituting (4.3) into (3.3) gives the solution in the following form:

$$
\begin{aligned}
u(x, t)= & \sum_{k=0}^{\infty} \sum_{h=0}^{\infty} U(k, h) x^{k} t^{h}=1+\left(x-\frac{x^{3}}{3 !}+\frac{x^{5}}{5 !}+\cdots\right) \\
& +\left(-\frac{1}{2}-\frac{3}{2} x-\frac{1}{2} x^{2}+\frac{1}{4} x^{3}+\frac{1}{6} x^{4}-\frac{1}{80} x^{5}+\cdots\right) t^{2} \\
& +\left(\frac{11}{24} x+\frac{1}{2} x^{2}+\frac{11}{144} x^{3}-\frac{1}{6} x^{4}-\frac{109}{2880} x^{5}+\cdots\right) t^{4}+\cdots
\end{aligned}
$$


Table 2: Comparison for the approximate values obtained by ADM, VIM, DTM, and PDTM at various values of $x$ and $t$.

\begin{tabular}{|c|c|c|c|c|}
\hline \multirow{2}{*}{$x$} & \multicolumn{4}{|c|}{$t=0.1$} \\
\hline & ADM & VIM & DTM & PDTM \\
\hline 0.2 & 1.190502988 & 1.190503087 & 1.190602734 & 1.190503088 \\
\hline 0.4 & 1.377844211 & 1.377844710 & 1.378073322 & 1.377844757 \\
\hline 0.6 & 1.549620480 & 1.549621939 & 1.550000812 & 1.549622051 \\
\hline 0.8 & 1.699081273 & 1.699084244 & 1.699640074 & 1.699084436 \\
\hline \multirow{2}{*}{$x$} & \multicolumn{4}{|c|}{$t=0.2$} \\
\hline & ADM & VIM & DTM & PDTM \\
\hline 0.2 & 1.166134875 & 1.166138050 & 1.166138150 & 1.166138093 \\
\hline 0.4 & 1.343423788 & 1.343432104 & 1.343438811 & 1.343435073 \\
\hline 0.6 & 1.505052082 & 1.505073495 & 1.505124251 & 1.505080633 \\
\hline 0.8 & 1.644954933 & 1.644997540 & 1.645259446 & 1.645009691 \\
\hline \multirow{2}{*}{$x$} & \multicolumn{4}{|c|}{$t=0.3$} \\
\hline & ADM & VIM & DTM & PDTM \\
\hline 0.2 & 1.125945576 & 1.125974851 & 1.125975665 & 1.125975548 \\
\hline 0.4 & 1.287943874 & 1.287088824 & 1.287130047 & 1.287122513 \\
\hline 0.6 & 1.432404521 & 1.432497282 & 1.432663372 & 1.432577763 \\
\hline 0.8 & 1.557040327 & 1.557215916 & 1.557830079 & 1.557352520 \\
\hline
\end{tabular}

The Projected DTM. The initial conditions $g_{i}(x), i=1,2$ yield $U(x, 0)=1+\sin x$ and $U(x, 1)=0$. Substituting $U(x, 0)$ and $U(x, 1)$ into (3.5) gives

$$
\begin{aligned}
u(x, t)= & \sum_{h=0}^{\infty} U(x, h) t^{h}=1+\sin x+\frac{1}{4}(-3+\cos 2 x-6 \sin x) t^{2} \\
& +\frac{1}{48}(12-12 \cos 2 x+25 \sin x-\sin 3 x) t^{4}+\cdots
\end{aligned}
$$

Table 2 shows the numerical results obtained by various methods. Here, the five terms of Adomian decomposition method (ADM), the fourth iteration of variational iteration method (VIM), the partial sum $S_{5,5}^{\mathrm{dtm}}$ of DTM, and the partial sum $S_{5}^{\mathrm{pdtm}}$ of PDTM are tested to compare with numerical results at various values of $x$ for each $t=0.1,0.2$, and 0.3 . For all test points $(x, t)$, numerical approximations obtained by the PDTM agree in three decimal places.

Example 4.2. Let us consider the nonlinear Klein-Gordon equation (3.1) with cubic nonlinearity $n=3$ with constants $\alpha=-1, \beta=1$ and $\gamma=1$ in the interval $(-1,1)$ [40]. The initial conditions are given by

$$
u(x, 0)=x^{2} \cosh x, u_{t}(x, 0)=x^{2} \sinh x
$$

That is, $g_{1}(x)=x^{2} \cosh x$ and $g_{2}(x)=x^{2} \sinh x$ in (3.2). The right-hand side function $f(x, t)$ in (3.1) is

$$
f(x, t)=\left(x^{2}-2\right) \cosh (x+t)-4 x \sinh (x+t)+x^{6} \cosh ^{3}(x+t)
$$


The Standard DTM. From the Taylor series expansion of $\sinh (x)$ and $\cosh (x)$, initial conditions $g_{i}(x), i=1,2$ give the following nonzero differential transforms $U(k, 0), U(k, 1)$, $k=0,1,2, \ldots$ :

$$
\begin{gathered}
U(2 k+2,0)=\frac{1}{(2 k) !} \\
U(2 k+3,1)=\frac{1}{(2 k+1) !} .
\end{gathered}
$$

Substituting (4.8) into (3.3) gives the solution in the following form:

$$
\begin{aligned}
u(x, t)= & \sum_{k=0}^{\infty} \sum_{h=0}^{\infty} U(k, h) x^{k} t^{h}=\left(x^{2}+\frac{x^{4}}{2}+\frac{x^{6}}{24}+\frac{x^{8}}{720}+\cdots\right) \\
& +\left(x^{3}+\frac{x^{5}}{6}+\frac{x^{7}}{120}+\cdots\right) t+\left(\frac{x^{2}}{2}+\frac{x^{4}}{4}+\frac{x^{6}}{48}+\cdots\right) t^{2}+\cdots
\end{aligned}
$$

The Projected DTM. The initial conditions $g_{i}(x), i=1,2$ yield $U(x, 0)=x^{2} \cosh (x)$ and $U(x, 1)=x^{2} \sinh (x)$. Substituting $U(x, 0)$ and $U(x, 1)$ into (3.5) gives

$$
\begin{aligned}
u(x, t)= & \sum_{h=0}^{\infty} U(x, h) t^{h}=x^{2} \cosh (x)+x^{2} \sinh (x) t+\frac{1}{2} x^{2} \cosh (x) t^{2} \\
& +\frac{1}{6} x^{2} \sinh (x) t^{3}+\frac{1}{24} x^{2} \cosh (x) t^{4}+\cdots
\end{aligned}
$$

In both methods, DTM and PDTM, the exact solution $u(x, t)$ can be obtained immediately from (4.9), (4.10) as

$$
u(x, t)=x^{2} \cosh (x+t)
$$

Table 3 shows the $L_{\infty}$ and $L_{2}$ error estimates of the numerical results obtained by the radial basis function method (RBF) [40], DTM, and PDTM at several values of $t$. In RBF, $\Delta t=0.0001$ and $\Delta x=0.01$ are used to obtain approximate solutions. In DTM and PDTM, the partial sums $S_{10,10}^{\mathrm{dtm}}$ and $S_{10}^{\mathrm{pdtm}}$ are tested. Since the DTM and PDTM are based on the Taylor series for the solution at $(x, t)=(0,0)$ and $t=0$, respectively, it is obvious that the more closer to $t=0$, the more accurate numerical approximation can be obtained. This can be shown in Table 3 . Moreover, the DTM and PDTM give inaccurate approximated solutions at $t=5$, but it can be easily improved by adding more terms in the partial sum. In fact, the partial sum $S_{20}^{\text {pdtm }}$ gives $1.4961 \times 10^{-5}$ in $L_{\infty}$ and $6.2667 \times 10^{-6}$ in $L_{2}$.

Example 4.3. Let us consider the following one-dimensional nonlinear Schrödinger equation (3.7) with $\alpha=1 / 2, \beta=\gamma=-1$ in the interval $(0,2 \pi)$ [41, 42]. Here, the trapping potential is $\psi(x)=\cos ^{2} x$ and the initial condition $u(x, 0)=\sin x$. 
Table 3: Comparison for the $L_{\infty}$ and $L_{2}$ error estimates of the approximate solutions obtained by RBF, DTM, and PDTM at each time.

\begin{tabular}{lccc}
\hline$t$ & & $L_{\infty}$ & PDTM \\
\hline 1 & RBF & DTM & $3.2870 \times 10^{-8}$ \\
2 & $5.0705 \times 10^{-5}$ & $3.7295 \times 10^{-6}$ & $7.5361 \times 10^{-5}$ \\
3 & $5.0260 \times 10^{-4}$ & $8.6492 \times 10^{-5}$ & $7.3229 \times 10^{-3}$ \\
4 & $2.0612 \times 10^{-3}$ & $7.3602 \times 10^{-3}$ & $1.9630 \times 10^{-1}$ \\
5 & $6.5720 \times 10^{-3}$ & $1.9639 \times 10^{-1}$ & $2.6048 \times 10^{0}$ \\
\hline$t$ & $1.9067 \times 10^{-2}$ & $2.6050 \times 10^{0}$ & PDTM \\
\hline 1 & & $L_{2}$ & $1.5413 \times 10^{-8}$ \\
2 & RBF & DTM & $3.2860 \times 10^{-5}$ \\
3 & $2.9474 \times 10^{-4}$ & $9.8387 \times 10^{-7}$ & $3.0333 \times 10^{-3}$ \\
4 & $2.7082 \times 10^{-3}$ & $3.5433 \times 10^{-5}$ & $7.8405 \times 10^{-2}$ \\
5 & $9.7246 \times 10^{-3}$ & $3.0402 \times 10^{-3}$ & $1.0170 \times 10^{0}$ \\
\hline
\end{tabular}

The Standard DTM. From the initial condition it is easy to obtain the following differential transforms $U(k, 0), k=0,1,2, \ldots$ :

$$
U(2 k+1,0)=\frac{(-1)^{k}}{(2 k+1) !}, \quad U(2 k, 0)=0
$$

Given trapping potential function $\psi(x)$ yields the nonzero differential transforms $\Psi(2 k, 0)$, $k=0,1, \ldots$ A few values of $\Psi(2 k, 0)$ are listed as follows:

$$
\Psi(2,0)=-1, \quad \Psi(4,0)=\frac{1}{3}, \quad \Psi(6,0)=-\frac{2}{45}, \ldots
$$

By substituting all coefficients $U(k, 0)$ and $\Psi(k, h)$ into (3.8), all values of $U(k, h)$ can be easily obtained. A few values of $U(k, h)$ are presented as follows:

$$
U(2 k, h)=0, \quad U(2 k+1,1)=-\frac{3 i}{2} \frac{(-1)^{k}}{(2 k+1) !}, \quad U(2 k+1,2)=-\frac{9}{8} \frac{(-1)^{k}}{(2 k+1) !}+\cdots
$$

Thus, we have

$$
\begin{aligned}
u(x, t)= & \sum_{k=0}^{\infty} \sum_{h=0}^{\infty} U(k, h) x^{k} t^{h}=\left(x-\frac{x^{3}}{6}+\frac{x^{5}}{120}-\cdots\right)-\frac{3 i}{2}\left(x-\frac{x^{3}}{6}+\frac{x^{5}}{120}-\cdots\right) t \\
& -\frac{9}{8}\left(x-\frac{x^{3}}{6}+\frac{x^{5}}{120}-\cdots\right) t^{2}+\frac{9 i}{16}\left(x-\frac{x^{3}}{6}+\frac{x^{5}}{120}-\cdots\right) t^{3}+\cdots
\end{aligned}
$$


The projected DTM. The initial condition $u(x, 0)$ gives $U(x, 0)=\sin (x)$ and the trapping potential function yields

$$
\Psi(x, h)= \begin{cases}\cos ^{2}(x), & h=0 \\ 0, & \text { otherwise }\end{cases}
$$

Substituting $U(x, 0)$ and $\Psi(x, h)$ into (3.9) gives

$$
u(x, t)=\sum_{h=0}^{\infty} U(x, h) t^{h}=\sin (x)-\frac{3 i}{2} \sin (x) t-\frac{9}{8} \sin (x) t^{2}+\frac{9 i}{16} \sin (x) t^{3}+\cdots
$$

From (4.15) and (4.17), the DTM and PDTM yield the following closed form:

$$
u(x, t)=\sin x \exp \left(-\frac{3 i}{2} t\right)
$$

which is the exact solution. Here, we compare the numerical results obtained by the spectral collocation method with preconditioning (SCMP) [41] and the proposed method. The test point $x_{i}$ is the Chebyshev-Gauss-Lobatto points in $(0,2 \pi) ; x_{i}=\pi \cos (i \pi / n)+\pi, n=16$. Suppose that the exact solution $u=v+w i$, then the absolute errors of the real and imaginary parts between the exact and approximation, $|E v|$ and $|E w|$, are defined by

$$
|E v(x, t)|=|v(x, t)-\widetilde{v}(x, t)|, \quad|E w(x, t)|=|w(x, t)-\widetilde{w}(x, t)|,
$$

where $\widetilde{v}, \widetilde{w}$ are the approximations obtained by numerical methods. Table 4 shows the absolute error estimates $|E v|$ and $|E w|$ at each test point $x_{i}$ for the fixed value $t=1$. In SCMP, $\Delta t=0.01$ and the partial sum $S_{15,15}^{\mathrm{dtm}}$ of DTM and the partial sum $S_{15}^{\mathrm{pdtm}}$ of PDTM are tested to obtain numerical approximations. It is shown that the standard DTM gives less accurate approximations at $x_{3}$ and $x_{7}$ compared with those obtained by PDTM. This is because the standard DTM is the Taylor series expansion at $(x, t)=(0,0)$ and $x_{3} \approx 5.75$ and $x_{7} \approx 3.75$ are far away from $x=0$. However, it does not occur in the PDTM at any value of $x_{i}$ because the PDTM depends on variable $t$, not space variable $x$.

Example 4.4. Let us consider the following two-dimensional nonlinear Schrödinger equation (3.7) with $\alpha=1 / 2, \beta=\gamma=-1$ in the interval $(0,2 \pi)^{2}[41,42]$. Here, the trapping potential is $\psi(x, y)=1-\sin ^{2} x \sin ^{2} y$ and the initial condition $u(x, y, 0)=\sin x \sin y$.

The Standard DTM. From the initial condition we have only nonzero $U(k, h, 0), k, h=$ $0,1,2, \ldots$ :

$$
U(2 k+1,2 h+1,0)=\frac{(-1)^{k+h}}{(2 k+1) !(2 h+1) !} .
$$


Table 4: Comparison for the absolute errors $|E v|$ and $|E w|$ of the approximate solutions obtained by SCMP, DTM, and PDTM at various test point $x_{i}$ with fixed time $t=1$.

\begin{tabular}{lccc}
\hline$x$ & & $\left|E v\left(x_{i}, 0\right)\right|$ & PDTM \\
\hline$x_{3}$ & SCMP & DTM & $1.5739 \times 10^{-11}$ \\
$x_{7}$ & $1.3281 \times 10^{-9}$ & $1.5029 \times 10^{-3}$ & $1.7926 \times 10^{-11}$ \\
$x_{11}$ & $2.2164 \times 10^{-9}$ & $1.1175 \times 10^{-6}$ & $3.0690 \times 10^{-11}$ \\
$x_{14}$ & $2.2164 \times 10^{-9}$ & $3.0748 \times 10^{-11}$ & $7.3817 \times 10^{-12}$ \\
\hline$x$ & $2.3774 \times 10^{-9}$ & $7.3818 \times 10^{-12}$ & PDTM \\
\hline$x_{3}$ & SCMP & $\left|E w\left(x_{i}, 0\right)\right|$ & $1.3898 \times 10^{-12}$ \\
$x_{7}$ & $9.4638 \times 10^{-10}$ & DTM & $1.5830 \times 10^{-12}$ \\
$x_{11}$ & $4.0901 \times 10^{-10}$ & $2.1193 \times 10^{-2}$ & $2.7101 \times 10^{-12}$ \\
$x_{14}$ & $4.0901 \times 10^{-10}$ & $1.5759 \times 10^{-5}$ & $6.5184 \times 10^{-13}$ \\
\hline
\end{tabular}

Given trapping potential function $\psi(x, y)$ gives the only nonzero $\Psi(2 k, 2 h, 0), k, h=0,1,2, \ldots$. A few values of $\Psi(2 k, 2 h, 0)$ are listed as follows:

$$
\begin{gathered}
\Psi(2,0,0)=-1, \quad \Psi(4,0,0)=\frac{1}{3}, \\
\Psi(2,2,0)=1, \quad \Psi(4,2,0)=-\frac{1}{3}, \quad \Psi(6,2,0)=\frac{2}{45} .
\end{gathered}
$$

Substituting all coefficients $U(k, h, 0)$ and $\Psi(k, h, 0)$ into (3.8) gives all values of $U(k, h, m)$. Table 5 lists the some values of the differential transform $U(k, h, m)$. Thus, we have

$$
\begin{aligned}
u(x, y, t)= & \sum_{k=0}^{\infty} \sum_{h=0}^{\infty} \sum_{m=0}^{\infty} U(k, h, m) x^{k} t^{h} t^{m} \\
= & \left(x-\frac{x^{3}}{6}+\frac{x^{5}}{120}-\cdots\right) y-\frac{1}{6}\left(x-\frac{x^{3}}{6}+\frac{x^{5}}{120}-\cdots\right) y^{3}+\cdots \\
& +\left\{-2 i\left(x-\frac{x^{3}}{6}+\frac{x^{5}}{120}-\cdots\right) y+\frac{i}{3}\left(x-\frac{x^{3}}{6}+\frac{x^{5}}{120}-\cdots\right) y^{3}+\cdots\right\} t \\
& +\left\{-2\left(x-\frac{x^{3}}{6}+\frac{x^{5}}{120}-\cdots\right) y+\frac{1}{3}\left(x-\frac{x^{3}}{6}+\frac{x^{5}}{120}-\cdots\right) y^{3}+\cdots\right\} t^{2}+\cdots
\end{aligned}
$$


Table 5: Some values of $U(k, h, m)$ in Example 4.4 .

\begin{tabular}{lrrl}
\hline$U(1,1,1)$ & $=-2 i$ & $U(1,1,2)=-2$ & $U(1,1,3)=\frac{4 i}{3}$ \\
$U(5,1,1)=-\frac{i}{60}$ & $U(5,1,2)=-\frac{1}{6}$ & $U(5,1,3)=\frac{i}{90}$ \\
$U(1,3,1)=\frac{i}{3}$ & $U(1,3,2)=\frac{1}{3}$ & $U(1,3,3)=-\frac{2 i}{9}$ \\
$U(5,3,1)=\frac{i}{360}$ & $U(5,3,2)=\frac{1}{360}$ & $U(5,3,3)=-\frac{i}{540}$ \\
$U(1,5,1)=-\frac{i}{60}$ & $U(1,5,2)=-\frac{1}{60}$ & $U(1,5,3)=\frac{i}{90}$ \\
$U(5,5,1)=-\frac{i}{7200}$ & $U(5,5,2)=-\frac{1}{7200}$ & $U(5,5,3)=\frac{i}{22680}$ \\
\hline
\end{tabular}

Table 6: Comparison for the $L_{\infty}$ errors between the exact solution and approximate solutions obtained by SSFD, DTM, and PDTM at $t=4$.

\begin{tabular}{lcc}
\hline SSFD & DTM & PDTM \\
\hline $8.115 \times 10^{-4}$ & $7.203 \times 10^{-4}$ & $7.203 \times 10^{-4}$ \\
\hline
\end{tabular}

The Projected DTM. The initial condition gives $U(x, y, 0)=\sin x \sin y$ and the trapping potential function yields $\Psi(x, y, 0)=1-\sin ^{2} x \sin ^{2} y$ and $\Psi(x, y, m)=0, m \neq 0$. Substituting $U(x, y, 0)$ and $\Psi(x, y, m)$ into (3.9) gives

$$
\begin{aligned}
u(x, y, t) & =\sum_{k=0}^{\infty} \sum_{h=0}^{\infty} \sum_{m=0}^{\infty} U(k, h, m) x^{k} y^{h} t^{m} \\
& =\left(1-2 i t-2 t^{2}+\frac{4 i}{3} t^{3}+\frac{2}{3} t^{4}-\frac{4 i}{15} t^{5}+\cdots\right) \sin x \sin y
\end{aligned}
$$

Both methods, DTM and PDTM, give directly the exact solution from (4.22) and (4.23):

$$
u(x, y, t)=\sin x \sin y \exp (-2 i t)
$$

Table 6 shows the $L_{\infty}$ error estimates between the exact and numerical solutions obtained by the split-step finite difference method (SSFD) [42] and the (P)DTM at $t=4$. In SSFD, $\Delta t=0.01$ is used to obtain approximated solutions. Since all numerical results are tested at $t=4$, a large number of partial sum in DTM and PDTM is considered. Here, the partial sum $S_{25,25,25}^{\mathrm{dtm}}$ of DTM and the partial sum $S_{25}^{\mathrm{pdtm}}$ are tested to obtain numerical approximations. It is worth noting that both DTM and PDTM yield accurate approximate solutions, different from the previous example. This is because of using the large number of partial sum in DTM. In other words, with 25-term partial sum the errors of approximate solutions obtained by DTM in the domain $(0,2 \pi)^{2}$ are almost negligible. 


\section{Conclusion}

In this work, we have developed the new modified version of differential transform method, the projected differential transform method, for solving the nonlinear Klein-Gorgon and Schrödinger equations. The PDTM uses the Taylor series on specific variable so that the corresponding algebraic equation is simple and easy to implement. It is concluded that, comparing with the standard DTM, the PDTM reduces computational cost in obtaining approximated solutions. Several illustrative examples are demonstrated to show the effectiveness for the PDTM. In all examples, the PDTM yields the exact solutions with simple calculation. Also, numerical results with partial sum in PDTM are compared with those obtained by various numerical methods such as ADM, VIM, RBF, SCMP, and SSFD. From all illustrative examples, it is shown that the PDTM yields very accurate approximate solutions. Thus, it is concluded that the PDTM is a powerful tool for solving linear and nonlinear problems. Here, all algebraic computations are performed by using Mathematica 7.0.

\section{Acknowledgments}

B. Jang is supported by the Basic Science Research Program through the National Research Foundation of Korea (NRF) funded by the Ministry of Education, Science and Technology (no. 2010-0013297), and Y. Do is supported by the WCU (World Class University) Program through the Korea Science and Engineering Foundation funded by the Ministry of Education, Science and Technology (no. R32-2009-000-20021-0).

\section{References}

[1] R. Hirota, "A new form of böcklund transformations and its relation to the inverse scattering problem," Progress of Theoretical Physics, vol. 52, no. 5, pp. 1498-1512, 1974.

[2] V. B. Matveev and M. A. Salle, Darboux Transformations and Solitons, Springer, Berlin, Germany, 1991.

[3] A. Chen and X. Li, "Darboux transformation and soliton solutions for Boussinesq-Burgers equation," Chaos, Solitons \& Fractals, vol. 27, no. 1, pp. 43-49, 2006.

[4] A.-M. Wazwaz, "The tanh method for compact and noncompact solutions for variants of the KdVBurger and the $K(n, n)$-Burger equations," Physica D, vol. 213, no. 2, pp. 147-151, 2006.

[5] M. Wang, Y. Zhou, and Z. Li, "Application of a homogeneous balance method to exact solutions of nonlinear equations in mathematical physics," Physics Letters, Section A, vol. 216, no. 1-5, pp. 67-75, 1996.

[6] S. Liu, Z. Fu, S. Liu, and Q. Zhao, "Jacobi elliptic function expansion method and periodic wave solutions of nonlinear wave equations," Physics Letters. A, vol. 289, no. 1-2, pp. 69-74, 2001.

[7] H. Zhang, "New exact Jacobi elliptic function solutions for some nonlinear evolution equations," Chaos, Solitons and Fractals, vol. 32, no. 2, pp. 653-660, 2007.

[8] J. F. Zhang, C. Q. Dai, Q. Yang, and J. M. Zhu, "Variable-coefficient F-expansion method and its application to nonlinear Schrodinger equation," Optics Communications, vol. 252, no. 4-6, pp. 408-421, 2005.

[9] M. A. Abdou, "The extended F-expansion method and its application for a class of nonlinear evolution equations," Chaos, Solitons and Fractals, vol. 31, no. 1, pp. 95-104, 2007.

[10] Sirendaoreji, "A new auxiliary equation and exact travelling wave solutions of nonlinear equations," Physics Letters, Section A, vol. 356, no. 2, pp. 124-130, 2006.

[11] B. Jang, "New exact travelling wave solutions of Kawahara type equations," Nonlinear Analysis: Theory, Methods \& Applications, vol. 70, no. 1, pp. 510-515, 2009.

[12] B. Jang, "New exact travelling wave solutions of nonlinear Klein-Gordon equations," Chaos, Solitons and Fractals, vol. 41, no. 2, pp. 646-654, 2009.

[13] G. Adomian, Solving Frontier Problems of Physics: The Decomposition Method, vol. 60, Boston, Mass, USA, 1994. 
[14] A.-M. Wazwaz, "Exact solutions to nonlinear diffusion equations obtained by the decomposition method," Applied Mathematics and Computation, vol. 123, no. 1, pp. 109-122, 2001.

[15] A. Sadighi and D. D. Ganji, "Analytic treatment of linear and nonlinear Schrödinger equations: a study with homotopy-perturbation and Adomian decomposition methods," Physics Letters A, vol. 372, no. 4, pp. 465-469, 2008.

[16] B. Jang, "Two-point boundary value problems by the extended Adomian decomposition method," Journal of Computational and Applied Mathematics, vol. 219, no. 1, pp. 253-262, 2008.

[17] J.-H. He, "Homotopy perturbation technique," Computer Methods in Applied Mechanics and Engineering, vol. 178, no. 3-4, pp. 257-262, 1999.

[18] J. H. He, "Application of homotopy perturbation method to nonlinear wave equations," Chaos, Solitons and Fractals, vol. 26, no. 3, pp. 695-700, 2005.

[19] J. Biazar and H. Ghazvini, “Exact solutions for non-linear Schrödinger equations by He's homotopy perturbation method," Physics Letters A, vol. 366, no. 1-2, pp. 79-84, 2007.

[20] A. Yildirim, "An algorithm for solving the fractional nonlinear Schrodinger equation by means of the homotopy perturbation method," International Journal of Nonlinear Sciences and Numerical Simulation, vol. 10, no. 4, pp. 445-450, 2009.

[21] J. H. He, "Variational iteration method-a kind of non-linear analytical technique: some examples," International Journal of Non-Linear Mechanics, vol. 34, no. 4, pp. 699-708, 1999.

[22] J.-H. He, "Variational iteration method—some recent results and new interpretations," Journal of Computational and Applied Mathematics, vol. 207, no. 1, pp. 3-17, 2007.

[23] T. Öziş and A. Yıldırım, "Application of He's semi-inverse method to the nonlinear Schrödinger equation," Computers $\mathcal{E}$ Mathematics with Applications, vol. 54, no. 7-8, pp. 1039-1042, 2007.

[24] A.-M. Wazwaz, "A study on linear and nonlinear Schrodinger equations by the variational iteration method," Chaos, Solitons and Fractals, vol. 37, no. 4, pp. 1136-1142, 2008.

[25] J. A. C. Weideman and B. M. Herbst, "Split-step methods for the solution of the nonlinear Schrödinger equation," SIAM Journal on Numerical Analysis, vol. 23, no. 3, pp. 485-507, 1986.

[26] P. Muruganandam and S. K. Adhikari, "Bose-Einstein condensation dynamics in three dimensions by the pseudospectral and finite-difference methods," Journal of Physics B, vol. 36, no. 12, pp. 2501-2513, 2003.

[27] V. M. Pérez-García and X.-y. Liu, "Numerical methods for the simulation of trapped nonlinear Schrödinger systems," Applied Mathematics and Computation, vol. 144, no. 2-3, pp. 215-235, 2003.

[28] J. K. Zhou, Differential Transformation and Its Application For Electrical Circuits, Huazhong University Press, Wuhan, China, 1986.

[29] M.-J. Jang, C.-L. Chen, and Y.-C. Liy, “On solving the initial-value problems using the differential transformation method," Applied Mathematics and Computation, vol. 115, no. 2-3, pp. 145-160, 2000.

[30] M.-J. Jang, C.-L. Chen, and Y.-C. Liu, "Two-dimensional differential transform for partial differential equations," Applied Mathematics and Computation, vol. 121, no. 2-3, pp. 261-270, 2001.

[31] I. H. Abdel-Halim Hassan, "Different applications for the differential transformation in the differential equations," Applied Mathematics and Computation, vol. 129, no. 2-3, pp. 183-201, 2002.

[32] F. Ayaz, "Solutions of the system of differential equations by differential transform method," Applied Mathematics and Computation, vol. 147, no. 2, pp. 547-567, 2004.

[33] A. Kurnaz, G. Oturanç, and M. E. Kiris, "n-dimensional differential transformation method for solving PDEs," International Journal of Computer Mathematics, vol. 82, no. 3, pp. 369-380, 2005.

[34] F. Kangalgil and F. Ayaz, "Solitary wave solutions for the KdV and mKdV equations by differential transform method," Chaos, Solitons and Fractals, vol. 41, no. 1, pp. 464-472, 2009.

[35] A. S. V. Ravi Kanth and K. Aruna, "Differential transform method for solving the linear and nonlinear Klein-Gordon equation," Computer Physics Communications, vol. 180, no. 5, pp. 708-711, 2009.

[36] B. Jang, "Comments on "Solving a class of two-dimensional linear and nonlinear Volterra integral equations by the differential transform method," Journal of Computational and Applied Mathematics, vol. 233, no. 2, pp. 224-230, 2009.

[37] B. Jang, "Solving linear and nonlinear initial value problems by the projected differential transform method," Computer Physics Communications, vol. 181, no. 5, pp. 848-854, 2010.

[38] Z. M. Odibat, C. Bertelle, M. A. Aziz-Alaoui, and G. H. E. Duchamp, "A multi-step differential transform method and application to non-chaotic or chaotic systems," Computers $\mathcal{E}$ Mathematics with Applications, vol. 59, no. 4, pp. 1462-1472, 2010.

[39] Y. Do and B. Jang, "Enhanced multistage differential transform method: application to the population models," Abstract and Applied Analysis, vol. 2010, Article ID 253890, 2012.

[40] M. Dehghan and A. Shokri, "Numerical solution of the nonlinear Klein-Gordon equation using radial 
basis functions," Journal of Computational and Applied Mathematics, vol. 230, no. 2, pp. 400-410, 2009.

[41] M. Javidi and A. Golbabai, "Numerical studies on nonlinear Schrödinger equations by spectral collocation method with preconditioning," Journal of Mathematical Analysis and Applications, vol. 333, no. 2, pp. 1119-1127, 2007.

[42] H. Wang, "Numerical studies on the split-step finite difference method for nonlinear Schrödinger equations," Applied Mathematics and Computation, vol. 170, no. 1, pp. 17-35, 2005. 


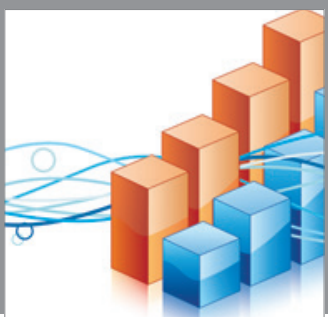

Advances in

Operations Research

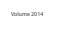

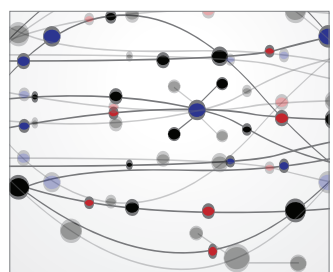

\section{The Scientific} World Journal
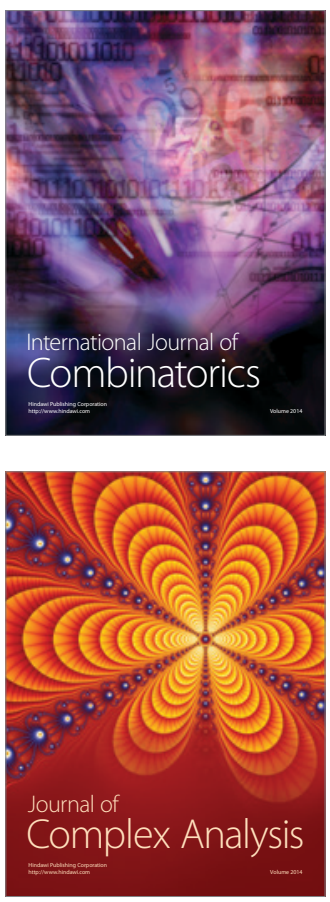

International Journal of

Mathematics and

Mathematical

Sciences
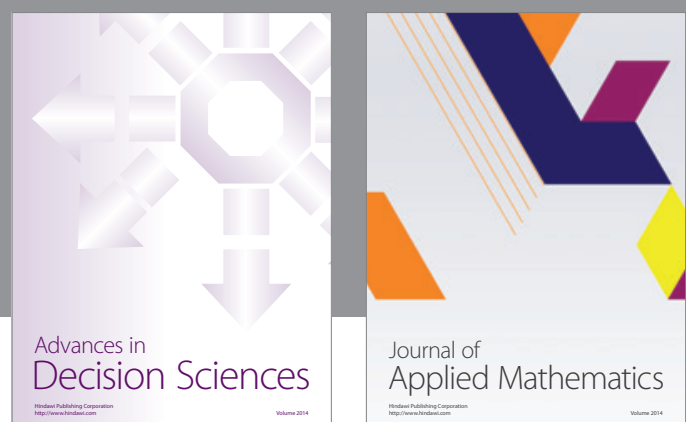

Journal of

Applied Mathematics
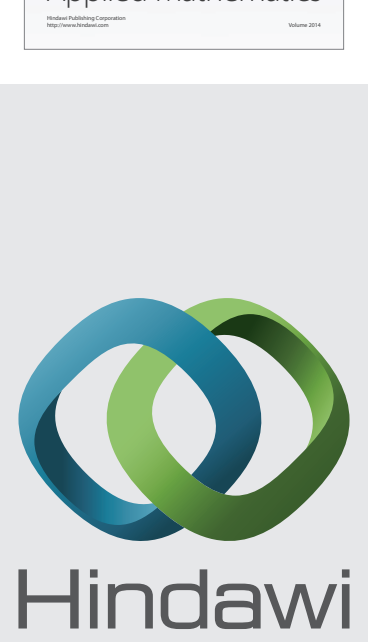

Submit your manuscripts at http://www.hindawi.com
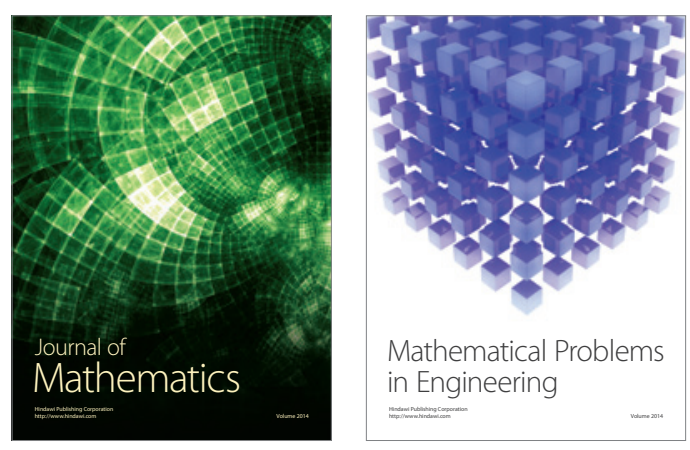

Mathematical Problems in Engineering
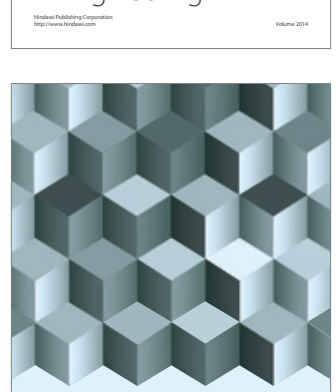

Journal of

Function Spaces
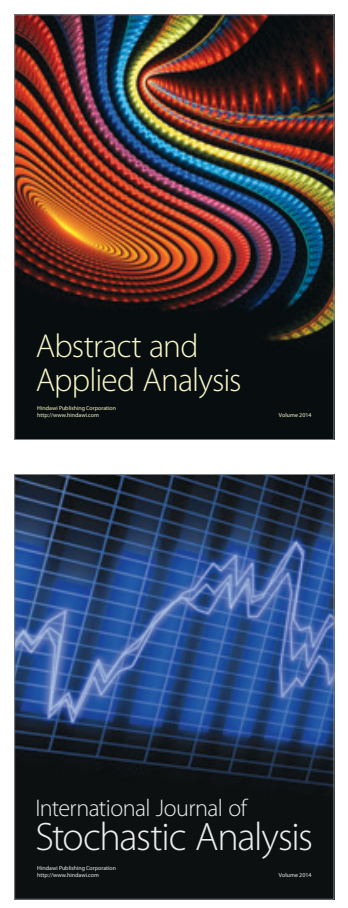

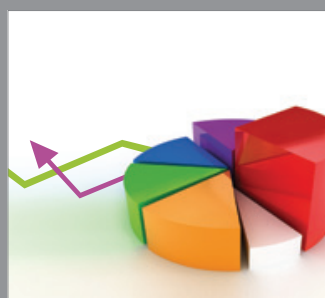

ournal of

Probability and Statistics

Promensencen
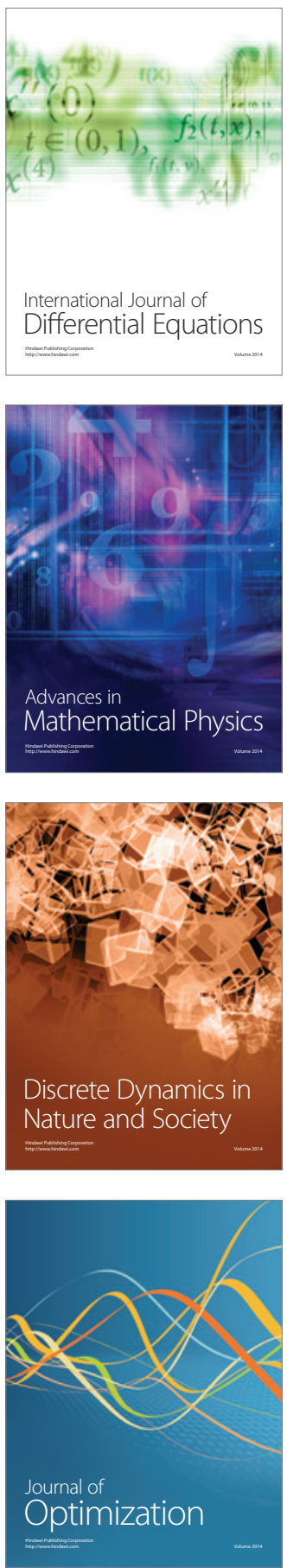